Research Article

\title{
Molecular cloning and expression analysis of a zebrafish novel zinc finger protein gene rnf141
}

\author{
Wenqian Deng, Huaqin Sun, Yunqiang Liu, Dachang Tao, Sizhong Zhang and Yongxin Ma \\ Department of Medical Genetics, West China Hospital \& Division of Morbid Genomics, State Key \\ Laboratory of Biotherapy, Sichuan University, Chengdu, Sichuan, People's Republic Of China.
}

\begin{abstract}
ZNF230 is a novel zinc finger gene cloned by our laboratory. In order to understand the potential functions of this gene in vertebrate development, we cloned the zebrafish orthologue of human ZNF230, named rnf141. The cDNA fragment of rnf141 was obtained by rapid amplification of cDNA ends (RACE). The open reading frame (ORF) encodes a polypeptide of 222 amino acids which shares $75.65 \%$ identity with the human ZNF230. RT-PCR analysis in zebrafish embryo and adult tissues revealed that $r n f 141$ transcripts are maternally derived and that $r n f 141 \mathrm{mRNA}$ has a broad distribution. Zygotic rnf141 message is strongly localized in the central nervous system, as shown by whole-mount in situ hybridization. Knockdown and over expression of rnf141 can induce abnormal phenotypes, including abnormal development of brain, as well as yolk sac and axis extendsion. Marker gene analysis showed that rnf141 may play a role in normal dorsoventral patterning of zebrafish embryos, suggesting that rnf141 may have a broad function during early development of vertebrates.
\end{abstract}

Key words: rnf141, zebrafish (Danio rerio), development, zinc finger protein.

Received: October 20, 2008; Accepted: May 13, 2009.

\section{Introduction}

The zinc finger gene family, one of the largest gene families in mammals, is defined by a conserved cysteine and histidine rich domain essential for the binding of zinc ions (Freemont 1993; Klug and Schwabe, 1995). This gene family can be divided into several subfamilies, including ring finger, $\mathrm{C} 2 \mathrm{H} 2$, glucocorticoid receptor, GATA1, GAL4, and LIM (Barlow et al., 1994; Borden and Freemont, 1996; Hammarstrom et al., 1996).

In accordance with their diverse structures, zinc finger proteins have been assigned multiple functions, including DNA recognition, transcriptional activation, RNA packaging, regulation of apoptosis, ubiquitination and many others (Coleman, 1992; Wolfe et al., 2000; Laity et al., 2001; Vazquez et al., 2007). More than 20 different zinc finger genes located on sex chromosomes or autosomes have been proposed to play a regulatory role in mammalian spermatogenesis (Noce et al., 1992; Pieler and Bellefroid, 1994; Yan et al., 2002).

The human ZNF230, which maps to the short arm of chromosome 11 (11p15), encodes a C3HC4-type zinc fin-

Send correspondence to Yongxin Ma. Department of Medical Genetics, West China Hospital \& Division of Morbid Genomics, State Key Laboratory of Biotherapy, Sichuan University, Renmin Nanlu, Section 3 \#17, Chengdu, Sichuan, People's Republic Of China. E-mail: mayongxin@gmail.com. ger protein motif (ring finger motif), and, consistent with a role in premeiotic or postmeiotic sperm development, one of its transcripts has been identified in abundance in the testicular tissue of fertile men, but neither in fetus nor in azoospermic patients. This suggested that ZNF230 may be involved in spermatogenesis, and loss of its expression may lead to azoospermia (Zhang et al., 2001). But so far, no clear biological function and mechanism had been elucidated.

In order to analyse the function of this novel zinc finger gene during vertebrate development, we decided to identify a potential orthologue in the teleost fish Danio rerio. This animal has become widely used as a genetic model to uncover specific functions of unknown proteins (Dooley and Zon, 2000; Rubinstein, 2003). Being transparent early embryonicl stages, easy to manipulate and highly reproductive makes the zebrafish an ideal animal system for molecular studies (Moro et al., 2007).

We here report the cloning and characterization of an 816 bp cDNA sequence, named $r n f 141$, which represents a candidate zebrafish orthologue of the human ZNF230 gene. By using whole-mount in situ hybridization, RT-PCR, gene knockdown and overexpression analysis, we further showed its spatiotemporal expression pattern during early developmental stages. 


\section{Materials and Methods}

\section{Zebrafish embryo maintenance}

Zebrafish $\mathrm{AB}$ strain was provided by National Zebrafish Resources of China, and maintained under standard laboratory conditions at $28.5^{\circ} \mathrm{C}$ (Westerfield, 1993). Embryonic stages were identified by morphological features (Kimmel et al., 1995), and embryos in developmental stages of interest were fixed in $4 \%$ paraformaldehyde.

\section{RNA extraction and reverse transcription}

Total RNAs was isolated from adult zebrafish tissues using RNeasy Mini Kit (Qiagen) according to the manufacturer's instructions. SuperScript TM Reverse Transcriptase (Invitrogen) was used for reverse transcription.

\section{Cloning of the zebrafish rnf141 gene}

Primers for 5'-RACE (366L 5'-ACGAGACGC CTCTACCATTCCATCC-3') and the other three pairs of primers (70U 5'-TCTCCATTTGGAGCCAAGATGGGC C-3' and 763L 5'- TAGATTTTTAAGGTCTGTGTGGG TG-3'; 338U 5'-AGGAGGATGGAATGGTAGAGGCGT C-3' and 619L 5'- GGCTCTGGCCGCTCCACTTGTCA AT-3'; and 432U 5'- TAGTTCAAATGTGGCGGCAG AGGGA-3' and 816L 5'-ATATATAGGTGGTCTTTT ATGGGGA-3') were designed to obtain the complete coding sequence, based on the potential orthologue of ZNF230 in zebrafish. This orthologue sequence was acquired by PSI-BLAST alignment. 5'-RACE experiments were performed using SMART RACE cDNA Amplification Kit (Clontech) (Frohman et al., 1988). cDNAs were reversetranscribed from total RNAs of zebrafish tissue. The PCR products, including 5'- RACE products, were ligated into the pGEM-T Easy Vector (Promega), cloned and sequenced bidirectionally. Sequences obtained by 5'-RACE and the other three fragments were assembled, and the contig was queried to the zebrafish genome database to determine its chromosomal location, and analyse its genomic structure. The deduced amino acid sequence was searched against InterPro Database for possible functional domains.

\section{Multi-tissue RT-PCR}

To reveal the tissue distribution and expression of zebrafish rnfl41 gene, total RNA was extracted from embryos of various developmental stages (Kimmel et al., 1995 ) and several tissues of adult zebrafish. The genespecific primers 488U 5'-GGATGGGCAGGGTAAAAC AGTTGA- 3'(forward) and 683L 5'-GCATCCGACATG ACCCAGGATTCATTAG-3' (reverse) were designed to amplify a $196 \mathrm{bp}$ fragment of rnf141. Amplification was performed in 30 cycles as follows: $30 \mathrm{~s}$ denaturation at $94{ }^{\circ} \mathrm{C}, 30$ s primer annealing at $62^{\circ} \mathrm{C}$ and 1 min extension at $72{ }^{\circ} \mathrm{C}$. The PCR products were electrophoresed on $1 \%$ agarose gel in $1 \mathrm{x}$ TAE buffer and ethidium bromide stained.
Primer sequences used for amplifying $470 \mathrm{bp} ß$-actin were 5'-TGTGGCCCTTGACTTTGAGCAG-3' (forward) and 5'-TAGAAGCATTTGCGGTGGACGA-3'(reverse), according to Kaslin et al., (2004),. In negative controls, $\mathrm{ddH}_{2} \mathrm{O}$ was used instead of cDNA template. The genespecific primers were selected from two exons separated by an intronic sequence to identify possible amplicons from contaminating genomic DNA. All synthetic oligonuleotides were purchased from Invitrogen Corporation (CA, USA).

\section{rnf141 gene knockdown and overexpression experiments}

rnf141 morpholino antisense oligonucleotide (rnf141-MO, 5'-CCAGAAAGCTGCTGGCCCATCTTG G-3') was used to target rnf141 mRNA, and 5'mispaired control morpholino (5'-CCAcAAAcCTcCTGcCCCATgT TGG-3') served as a control. Both were designed by using Gene-tools (Philomath, OR). The coding region of rnfl41 was ligated into vector pcDNA3 (Invitrogen) and linearized by appropriate restriction enzymes for mRNA was synthesis by using mMESSAGE mMACHINE® Kit (Ambion). rnf141-MO and control-MO were injected into 1-2 cell zebrafish embryos by using a Model PLI-90 Pico-Injector.

\section{Whole-mount in situ hybridization}

rnf141 sense and antisense RNA probes were labeled with digoxigenin-11-UTP and synthesized by using DIG RNA Labeling Kit (SP6/T7) (Roche). The template for the probe was the entire 668 bp long cDNA. Whole-mount in situ hybridizations was performed as described by Westerfield (1995). Images were captured using an Olympus digital camera.

\section{Results and Discussion}

\section{Zebrafish rnf141 has a C3HC4 zinc finger domain}

We cloned zebrafish $r n f 141$ based on the amino acid sequence of human ZNF230 and mouse znf230 by PSIBLAST alignment and RT-PCR including RACE. As a result, four fragments were obtained that formed a816 bp cDNA contig. This was submitted to GenBank (accession number: AY621088). Previous studies have shown that human ZNF230 has two transcripts (Zhang et al., 2001). In mouse, there are three transcripts of znf230 (Qiu et al. 2003). In contrast, in our analyses on zebrafish we found only one transcript of this gene. Furthermore, our result was confirmed by another mRNA sequence record (GenBank accession numbe $\mathrm{BC} 071534$ ) that encodes the same protein submitted by Strausberg et al.. Also, the sequence of our 3 '-RACE fragment is identical to this sequence record.

The predicted open reading frame from 88 to 756 is $668 \mathrm{bp}$ in length and encodes a polypeptide of 222 amino acid residues with a $\mathrm{C} 3 \mathrm{HC} 4$ zinc finger domain from 146 to 187 amino acid residues (Figure 1). Proteins with such a 
1 tนtgttaggataaaacaa tgccaacagggcctgcagtggtgaatgaggtctagagttaa 61 tagagcagctctccatt geagccaagATGGGCCAGCAGCTITCTGGCCAAGCAGTGACC 121 CGATTACCTGAGAAACTGATCAAACATGTGGGGCTGGTGCGGGACAGTGGATATCTCACC $\begin{array}{llllllllllllllllllllll}R & \text { L } & P & E & K & \text { L } & \text { I } & \text { K } & \text { H } & \text { V } & G & \text { L } & \text { V } & \text { R } & \text { D } & \text { S } & G & \text { Y } & \text { L } & \text { T }\end{array}$ TATGATGAGITCTTGGGTCGTGTGGCCGAGCTCAATGATGTAACTGCAAAACTCGCCTCT

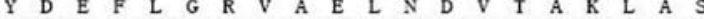
GGICAAAAAAACATCTATTGTITGAAGTGCAGCCTGGATCTGATTCCAGTGCTITGTGG $G$ Q $\quad K \quad K$ H $L$ AAAGTAGCAGTTAGAATAGTCTCCACCAAGATTAATAAGGAGCATGGAATGCTAGACGCG $\mathrm{K} V \mathrm{~V}, \mathrm{~V} R$ I V C T K I V K E D G VGIAGAGGCG TCTCGTATTATGAACTIGTACCAGITIATTCAGCTCTACAAAGACATCACCAGTCAAGCA

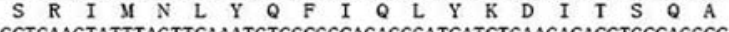
GCTGAAGTATTTAGTTCAAATGTGGCGGCAGAGGGATCATCTGAAGACACCTGCCAGGCC $\begin{array}{llllllllllllllllllll}A & E & V & F & S & S & N & V & A & A & E & G & S & S & E & D & T & C & Q & A\end{array}$ AGCATGTGGATGGGCAGGGTAAAACAGTTGACTGATGAGGAGAATGCTGTATCTGCATG S M M $\quad$ W GATGGGAAAGCAGATCTITATTCTICCCTGTGCGCACAGCTTCTGTCAGAAGTGTATTGAC $\begin{array}{lllllllllllllllllllll}D & G & K & A & D & \text { L } & \text { I } & \text { L } & \text { P } & \text { C } & \text { A } & \text { H } & \text { S } & \text { F } & \text { C } & \text { Q } & \text { K } & \text { C } & \text { I } & \text { D }\end{array}$ AAGTGGAGCGGCCAGAGCCGAAACTGCCCCGTCTGCCGGATTCAGGTGACTGCCGCTAAT

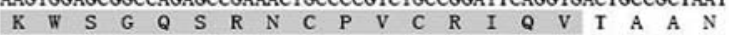
GAATCCTGGGTCATGTCGGATGCTCCCACAGGGGAAGATGTAGCCGGGTACATTCTCAAT

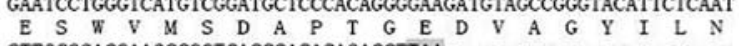
CTIGCGGACGAAGCCGGTCACCCACACAGACCIIAAaaatctaacact gtgccactctca

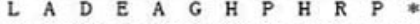

781 tgctcaccatctcccataaaagaccacctatatat

Figure 1 - Nucleotide and predicted amino acid sequence of $r n f 141$ gene. The coding region (nucleotides 88-756) is in uppercase letters. The translation initiation codon is underlined. The stop codon at the 3'-end of the sequence is underlined and shaded. The deduced amino acid sequence (222 amino acids) is also shown below the nucleotide sequence. The predicted Ring-finger motif is shaded.

structure generally are nuclear transcriptional factors with the motifs being involved in both protein-DNA and protein-protein interactions. We found that zebrafish rnf141 protein shares $75.65 \%$ and $75.22 \%$ identity in amino acid sequence with the human and mouse homologues, respectively. Furthermore, aligning amino acid sequence of zebrafish rnfl41 with ZNF230 of other vertebrate species indicated that this gene domain is highly conserved (date not shown), suggesting functional similarity and conservation.

\section{rnf141 is expressed in the CNS, primarily during} early embryogenesis

To analyse the spatiotemporal expression of rnfl41 during early embryonic development, whole-mount in situ hybridizations were performed on two-cell stage to fiveday-old embryos using an antisense probe. As a result, rnf141 transcripts were already detected at the two-cell stage (Figure 2A), thus suggesting a maternal origin of the transcript. From the sphere stage $(4 \mathrm{hpf})$ to the tail bud stage (10 hpf) (Figure 2C-E), the rnfl 41 transcripts have a broad distribution. However, at the 5-somite stage (11.6 hpf), a characteristic pattern was displayed with marked staining in the notochord (Figure 2F), and at the Prim-5 stage of the pharyngula period, this pattern was displayed in the midbrain and hindbrain (Figure 2G). Following the long-pec stage (48 hpf), restricted signal localization was evident in the otic capsule, 4th ventricle, epiphysis and cerebellum (Figure 2H-I). When embryos reached the protrudingmouth stage (72 hpf), obvious signals were detected in the oral cavity and otic capsule (Figure 2J-K). In $5 \mathrm{dpf}$ (120 hpf) embryos, an extensive rnfl 41 expression was visible in the gut, with a restricted localization in the swim bladder (Figure 2L). To assess the specificity of the antisense probe, a sense probe was used in a parallel control experiment at all stages. With this sense probe no staining was detected in any embryo (Figure 3).

The consistency of hybridization experiments was confirmed by RT-PCR expression analysis performed on cDNAs from whole zebrafish embryos at various early developmental stages (Figure $2 \mathrm{M}$ ).

Since the $1 \mathrm{~kb}$ transcript of human ZNF230 is only expressed in fertile male testis, whereas another $4.4 \mathrm{~kb}$ transcript was detected in many tissues; include heart, brain, skeletal muscle, kidney and pancreas (Zhang et al., 2001), we further addressed the question as to whether zebrafish rnf141 maintains its ubiquitous spatial expression in adult stages. As shown in Figure 2N, RT-PCR based analysis demonstrated that almost all analysed tissues of adult fish do display a high content of rnfl41 transcripts.

In conclusion, these results of whole-mount in situ hybridization and RT-PCR analyses performed both on zebrafish embryos and adult tissues provide evidence that rnf141 may have multiple functions. The detection of its transcripts in the CNS of early embryos, especially restricted in the notochord at the 5-somite of the segmentation period, suggests a function for rnfl41 in zebrafish development. Further analysis is ongoing in order to improve knowledge on the role of $r n f 141$.

\section{rnf141 may play a part in normal dorsoventral patterning of zebrafish embryos}

To further study the potential function of $r n f 141$, we first injected zebrafish embryos with synthetic rnfl41 mRNA. Injection of $200 \mathrm{pg}$ rnfl41 mRNA caused $84 \%$ $(n=92)$ of the embryos to show phenotypes that are characteristic of embryonic ventralization at $24 \mathrm{hpf}$ (Figure 4B). The expression of the shield-specific gene goosecoid was decreased at the shield stage (Figure 5Ac"). In contrast, the ventral markers $b m p 2 b$ and vent expanded dorsally during gastrulation (Figure 5Ac-c'). The ratios of embryos with altered marker gene expression are summarized in Figure 5B.

To investigate the role of endogenous rnfl41, a morpholino antisense oligonucleotide ( $r n f 141-\mathrm{MO})$ was injected into one-cell embryos. As a result, $81 \%(n=88)$ of the embryos injected with $12 \mathrm{ng}$ rnfl41-MO exhibited dorsalized phenotypes at $24 \mathrm{hpf}$ : complete loss of the yolk sac extension and partial loss of the caudal ventral fin (Figure 4C). The effects of rnfl41 knockdown on the expression of the marker genes bmp2b, vent and goosecoid (Figure 5Ab-b") tended to be opposite to those of rnfl41 overexpression. In contrast, injection with $15 \mathrm{ng}$ of control morpholino, which differs from $r n f 141$-MO in five mismatched nucleotides, did not cause developmental defects (Figure 4E).

To test the efficiency of the morpholinos, fertilized eggs were injected with $12 \mathrm{ng}$ rnfl41-MO in combination with $100 \mathrm{pg}$ of prnf141-GFP DNA, an expression construct 
containing the full coding region of rnfl 41 cDNA fused in-frame to a GFP coding sequence. At this dose of rnfl41-
$\mathrm{MO}$, the injected embryos almost lacked green fluorescence from the GFP fusion protein (Figure 5I), while the

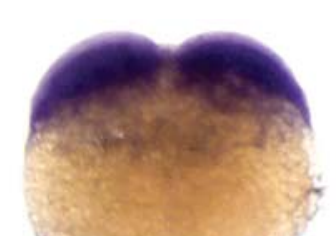

$A=B$

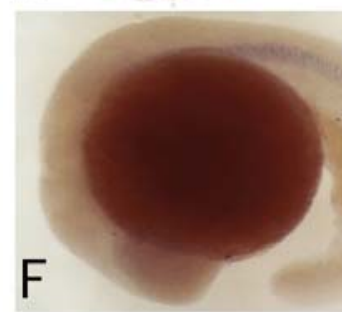

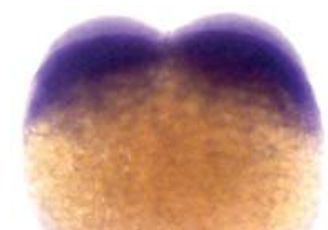

B

nc

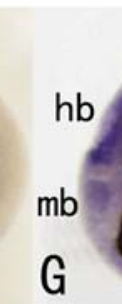

C

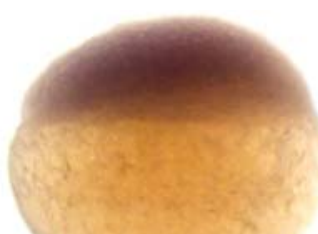

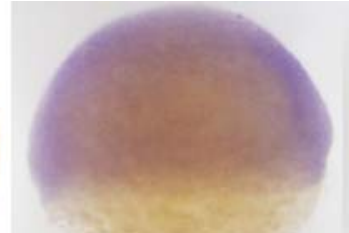

D

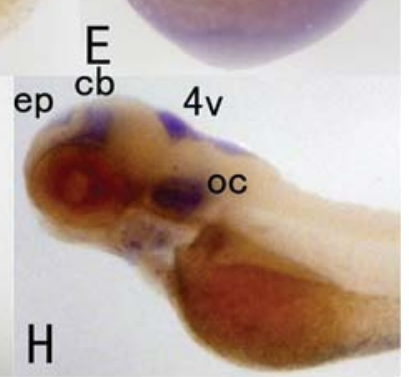

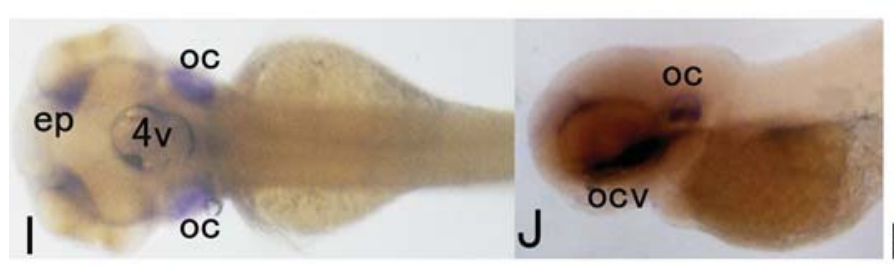
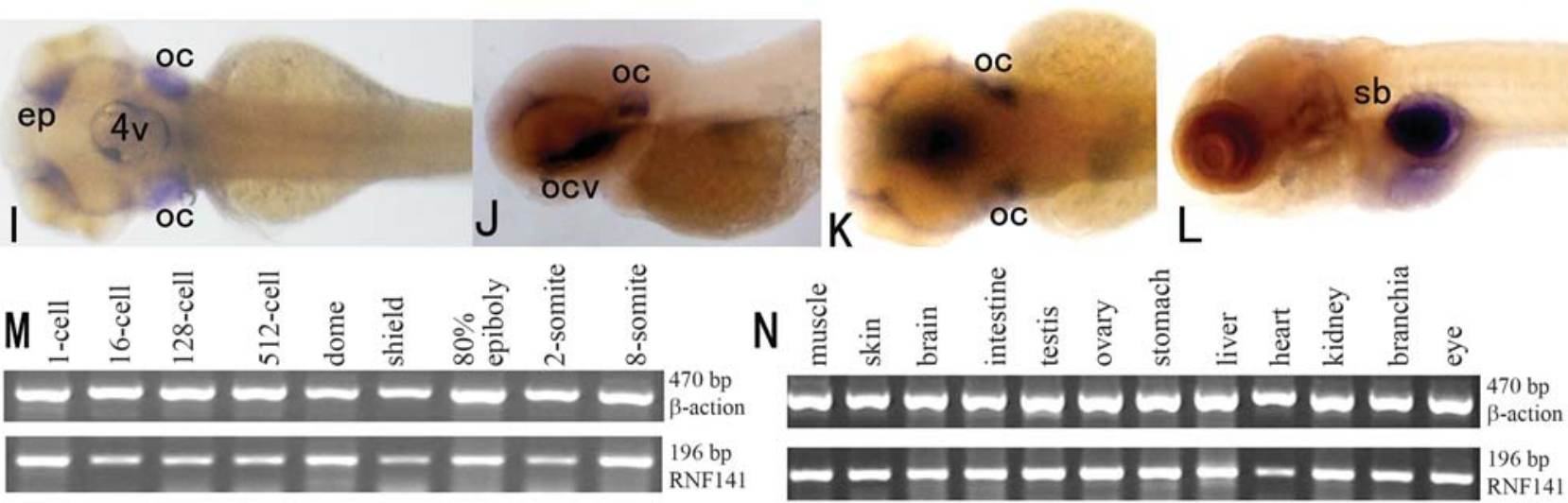

Figure 2 - Expression analysis of rnf141 in early embryos and adult zebrafish tissues. rnf141 mRNA was initially detected at the 2-cell stage (A) and 4-cell stage (B), the signal become weaker at the sphere stage (C), shield stage (D) and bud stage (E). At the 5-somite stage (11.6 hpf), a characteristic pattern was displayed with marked staining in the notochord (F). At the 5-prim stage (24 hpf), a strong signal was detected in the head, particularly in the midbrain, hindbrain and the otic capsule (G). At the long-pec stage ( $48 \mathrm{hpf}$ ) the signal localization became restricted to the otic capsule, the $4^{\text {th }}$ ventricle, as well as the epiphysis and tegmentum (H-lateral view from left; I-dorsal view). An even more restricted expressions was detected in the oral cavity and otic capsule when embryos reached the mouth-protruding stage ( $72 \mathrm{hpf}$ ) (J- lateral view from left; K-dorsal view). An extensive expression in the gut and a restricted localization in swim bladder was found in $5 \mathrm{dpf}$ embryo (L). Expression analysis of rnfl 141 detected by RT-PCR in different developmental stage embryos (M) and adult zebrafish tissues (N). Abbreviations: mb, midbrain; hb, hindbrain; ep, epiphysis; cb, cerebellum; 4v, 4th ventricle; oc, otic capsule; ocv, oral cavity; sb, swim bladder.

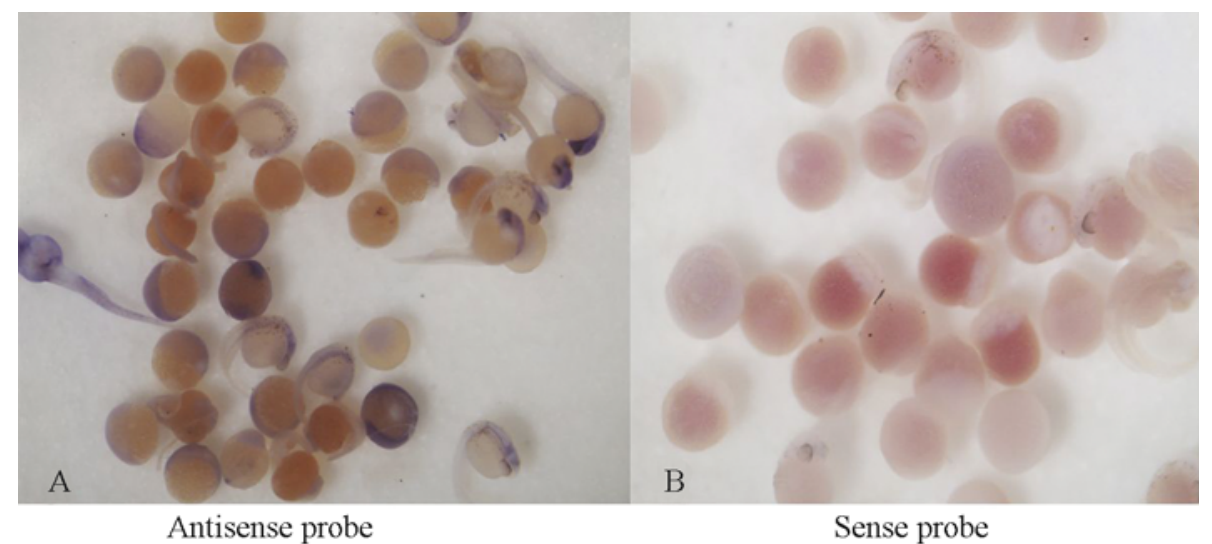

Figure 3 - Whole-mount in situ hybridization with rnf141 antisense and sense probe. Hybridizations were performed on two-cell stage to five-day-old embryos using rnf141 antisense probe (A) and sense probe (B). 

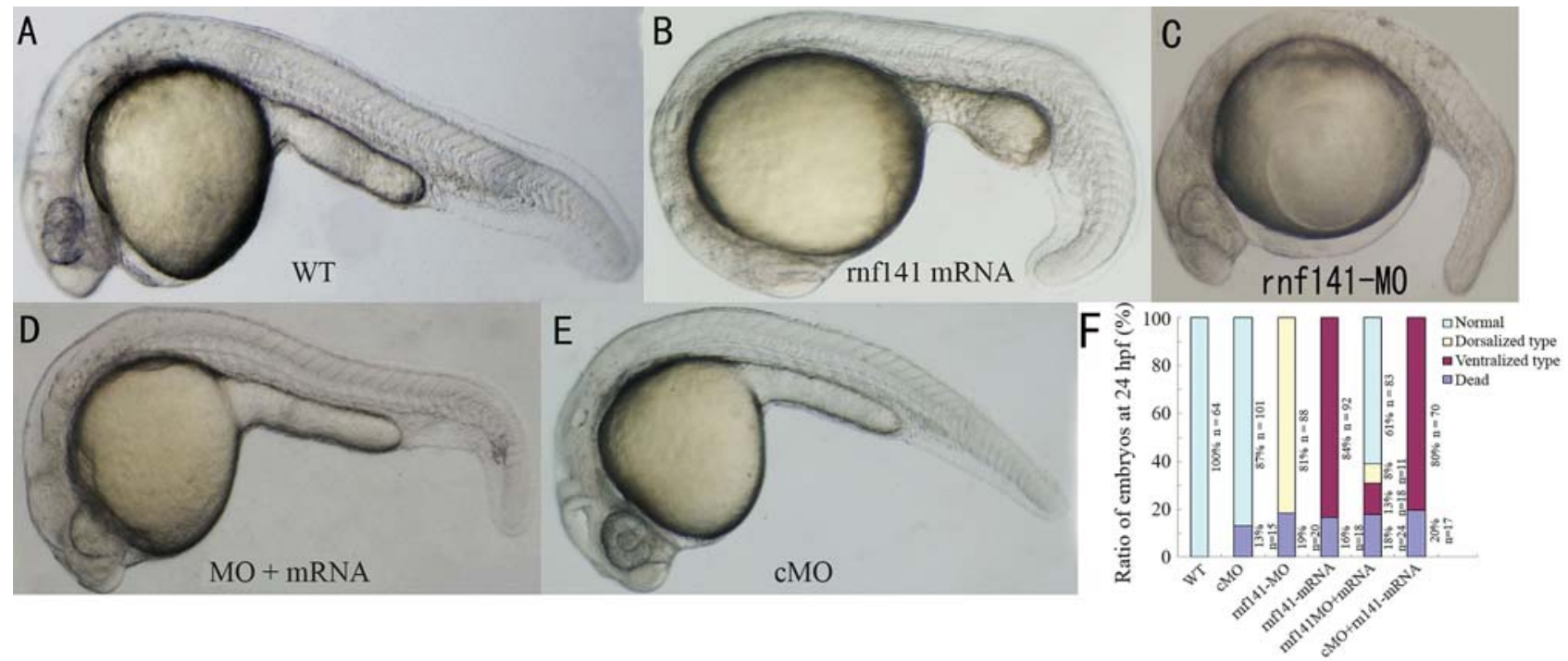

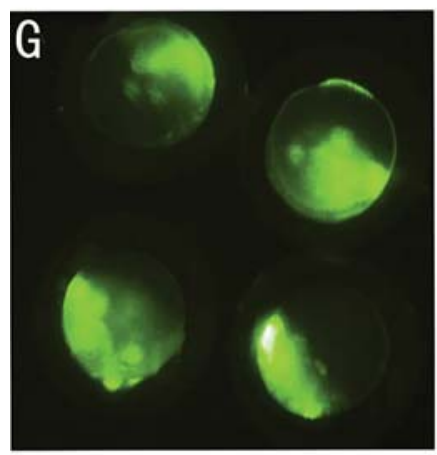

rnf141-GFP

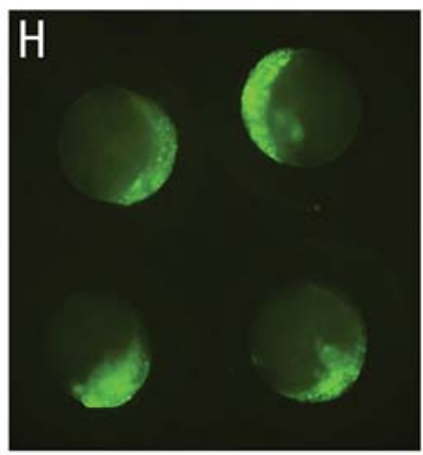

rnf141-GFP + cMO

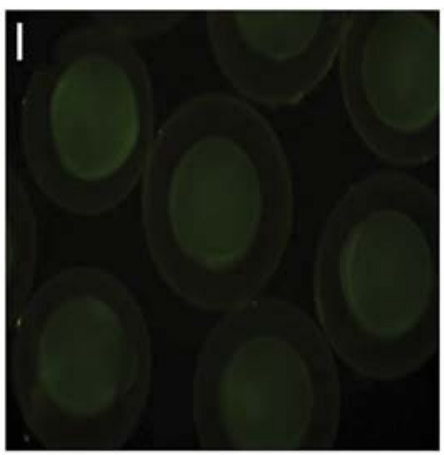

rnfl41-GFP + rnfl41-MO

Figure 4 - Knockdown and overexpression analysis of $r n f 141$. All images are lateral views of live embryos at 24 hpf, anterior is to the left. (A) Wild-type embryo. (B) Injection with $200 \mathrm{pg}$ rnf141 mRNA resulted in enlargement of the yolk sac, in addition an extension and broadening of caudal ventral fin. (C) Injection with $12 \mathrm{ng}$ rnfl41-MO led to caudal ventral fin loss of and yolk sac extension. (D) Coinjection with 100 pg rnfl41 mRNA and 12 ng

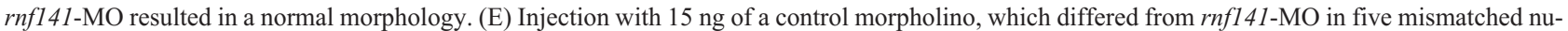
cleotides, did not show visible developmental defects. (F) The ratios of embryos showing different phenotype in experiments represented in A-E. Data were averaged from three independent experiments and expressed as means and standard deviations. The numbers of analysed embryos are indicated beneath each bar. (G-I) Live embryos at the shield stage, (G) embryos injected with 100 pg prnf141-GFP DNA. (H) Embryos injected with 100 pg prnf141-GFP DNA and 12 ng rnf141-5mis-MO. (I) embryos injected with 100 pg prnf141-GFP DNA and 12 ng rnf141-MO.

same dose of rnf141-cMO injected embryos retained visible fluorescence (Figure 5H), suggesting that rnf141-MO could effectively block translation of rnfl41 mRNA.

To test the specificity of rnfl41-MO, a 5 mis-pair rnf141 mRNA corresponding to 5 mis-pair control morpholino was synthesized for rescuing the phenotype mediated with rnfl41-MO. These results showed that the rnf141-MO-induced dorsalization could be neutralized by coinjection with a smaller amount of 5 mis-pair rnf141 (Figure 4D), suggesting that rnf141-MO specifically targets $r n f 141$.

In conclusion, knockdown of $r n f 141$ by using special morpholino-induced abnormal outcomes, including inordinate development of the CNS with an atrophic hindbrain, thin and crooked notochord, as well as disappearance of yolk sac extension, abnormality of axis, and partial loss of the caudal ventral fin. These embryos are characteristic of weakly dorsalized phenotypes, reminiscent of mini fin ( $m f n$ ) and lost-a-fin (laf) mutant embryos, which were first described by Mullins et al (1996) and were subsequently found to be caused by inefficient BMP signaling (Bauer et al., 2001; Connors et al., 1999; Mintzer et al., 2001). Overexpressing this gene by injection of rnf141 mRNA may caused embryos to show ventralized phenotypes. We also noted that rnf141-MO-induced dorsalization could be neutralized by coinjection of a smaller amount of $r n f 141$ mRNA, suggesting that rnf141-MO specifically targets rnf141 mRNA. The expression of ventral markers ( bmp $2 b$ and vent) and of a dorsal marker (goosecoid) were impacted by altered expression of rnfl41, thus suggesting that zebrafish rnf141 may participate in normal dorsoventral embryonic patterning. Further research is needed to better understand the respective biological pathway(s) and improve the knowledge on the function of rnfl41. 

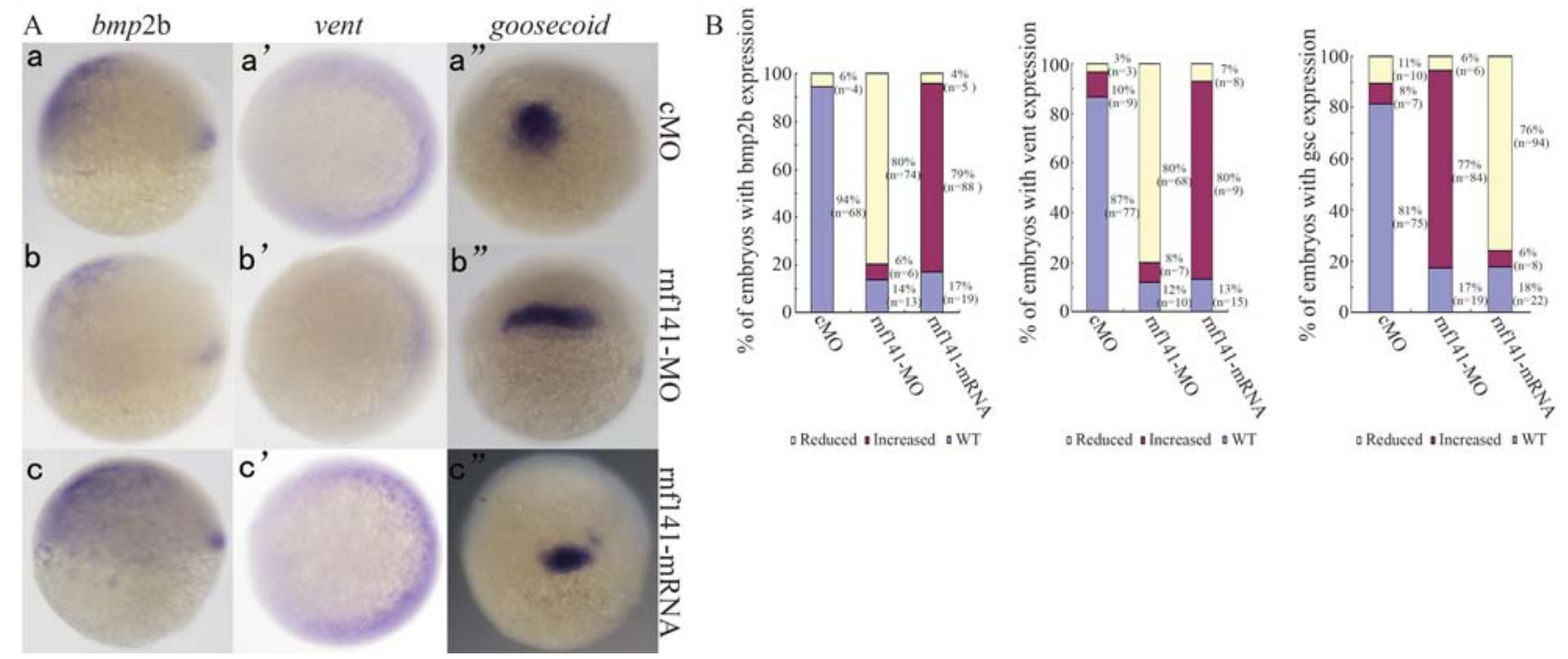

Figure 5 - Expression patterns of marker genes in injected embryos at shield stage. (A) Expression patterns of bmp $2 b$, vent, goosecoid in embryos injected with $15 \mathrm{ng}$ rnfl41-5mis-MO, $12 \mathrm{ng}$ rnfl41-MO, or $200 \mathrm{pg}$ rnfl $141 \mathrm{mRNA}$, respectively. For bmp2b expression, the embryo is shown in lateral view with dorsal pointed towards right; the embryo showing vent expression is depicted in animal pole view with dorsal oriented towards left; and the embryo with goosecoid expression is shown in a dorsal view with the animal pole pointed towards the top. (B) Statistical data for each marker gene of shown in A.

\section{Acknowledgments}

This research is supported by the National Natural Science Foundation of China (30770812, 90408025 and 30500186) and National High-Tech Research and Development Program of China (2008AA02Z102).

\section{References}

Barlow PN, Luisi B, Milner A, Elliott M and Everett R (1994) Structure of the $\mathrm{C} 3 \mathrm{HC} 4$ domain by $1 \mathrm{H}$-nuclear magnetic resonance spectroscopy. A new structural class of zincfinger. J Mol Biol 237:201-211.

Bauer H, Lele Z, Rauch GJ, Geisler R and Hammerschmidt M (2001) The type I serine/threonine kinase receptor Alk8/Lost-a-fin is required for Bmp2b/7 signal transduction during dorsoventral patterning of the zebrafish embryo. Development 128:849-858.

Borden KL and Freemont PS (1996) The RING finger domain: A recent example of a sequence-structure family. Curr Opin Struct Biol 6:395-401.

Coleman JE (1992) Zinc proteins: Enzymes, storage proteins, transcription factors, and replication proteins. Annu Rev Biochem 61:897-946.

Connors SA, Trout J, Ekker M and Mullins MC (1999) The role of tolloid/mini fin in dorsoventral pattern formation of the zebrafish embryo. Development 126:3119-3130.

Dooley K and Zon LI (2000) Zebrafish: A model system for the study of human disease. Curr Opin Genet Dev 10:252-256.

Freemont PS (1993) The RING finger. A novel protein sequence motif related to the zinc finger. Ann NY Acad Sci 684:174-192.

Frohman MA, Dush MK and Martin GR (1988) Rapid production of full-length cDNAs from rare transcripts: Amplification using a single gene-specific oligonucleotide primer. Proc Natl Acad Sci USA 85:8998-9002.
Hammarstrom A, Berndt KD, Sillard R, Adermann K and Otting G (1996) Solution structure of a naturally-occurring zincpeptide complex demonstrates that the $\mathrm{N}$-terminal zinc-binding module of the Lasp-1 LIM domain is an independent folding unit. Biochemistry 35:12723-12732.

Kaslin J, Nystedt JM, Ostergard M, Peitsaro N and Panula P (2004) The orexin/hypocretin system in zebrafish is connected to the aminergic and cholinergic systems. J Neurosci 24:2678-2689.

Kimmel CB, Ballard WW, Kimmel SR, Ullmann B and Schilling TF (1995) Stages of embryonic development of the zebrafish. Dev Dyn 203:253-310.

Klug A and Schwabe JW (1995) Protein motifs 5. Zinc fingers. FASEB J 9:597-604.

Laity JH, Lee BM and Wright PE (2001) Zinc finger proteins: New insights into structural and functional diversity. Curr Opin Struct Biol 11:39-46.

Mintzer KA, Lee MA, Runke G, Trout J, Whitman M and Mullins MC (2001) Lost-a-fin encodes a type I BMP receptor, Alk8, acting maternally and zygotically in dorsoventral pattern formation. Development 128:859-869.

Moro E, Maran C, Slongo ML, Argenton F, Toppo S and Onisto M (2007) Zebrafish spata2 is expressed at early developmental stages. Int J Dev Biol 51:241-246.

Mullins MC, Hammerschmidt M, Kane DA, Odenthal J, Brand M, van Eeden FJ, Furutani-Seiki M, Granato M, Haffter P, Heisenberg CP, et al. (1996) Genes establishing dorsoventral pattern formation in the zebrafish embryo: The ventral specifying genes. Development 123:81-93.

Noce T, Fujiwara Y, Sezaki M, Fujimoto H and Higashinakagawa $\mathrm{T}$ (1992) Expression of a mouse zinc finger protein gene in both spermatocytes and oocytes during meiosis. Dev Biol 153:356-367.

Pieler T and Bellefroid E (1994) Perspectives on zinc finger protein function and evolution - An update. Mol Biol Rep 20:1-8. 
Qiu W, Zhang S, Xiao C, Xu W, Ma Y, Liu Y and Wu Q (2003) Molecular cloning and characterization of a mouse spermatogenesis-related ring finger gene znf230. Biochem Biophys Res Commun 306:347-353.

Rubinstein AL (2003) Zebrafish: From disease modeling to drug discovery. Curr Opin Drug Discov Dev 6:218-223.

Strausberg RL, Feingold EA, Grouse LH, Derge JG, Klausner RD, Collins FS, Wagner L, Shenmen CM, Schuler GD, Altschul SF, et al. (2002) Generation and initial analysis of more than 15,000 full-length human and mouse cDNA sequences. Proc Natl Acad Sci USA 99:16899-16903.

Vazquez O, Vazquez ME, Blanco JB, Castedo L and Mascarenas JL (2007) Specific DNA recognition by a synthetic, monomeric Cys2His2 zinc-finger peptide conjugated to a minor-groove binder. Angew Chem Int Ed Engl 46:6886-6890.

Westerfield M (1993) The Zebrafish Book: A Guide for the Laboratory Use of Zebrafish. University of Oregon Press, Oregon, pp 8-48.

Westerfield M (1995) The Zebrafish Book: A Guide for the Laboratory Use of Zebrafish. Eugene Press, Oregon, pp 200-204.
Wolfe SA, Nekludova L and Pabo CO (2000) DNA recognition by Cys2His2 zinc finger proteins. Annu Rev Biophys Biomol Struct 29:183-212.

Yan W, Burns KH, Ma L and Matzuk MM (2002) Identification of Zfp393, a germ cell-specific gene encoding a novel zinc finger protein. Mech Dev 118:233-239.

Zhang S, Qiu W, Wu H, Zhang G, Huang M, Xiao C, Yang J, Kamp C, Huang X, Huellen K, et al. (2001) The shorter zinc finger protein ZNF230 gene message is transcribed in fertile male testes and may be related to human spermatogenesis. Biochem J 359:721-727.

\section{Internet Resources}

Zebrafish Genome Resources, http://www.ncbi.nlm.nih.gov/projects/genome/guide/zebrafish/ (U.S. National Library of Medicine, Maryland MD, 2005).

InterPro Database, http://www.ebi.ac.uk/interpro/ (European Bioinformatics Institute, 2006).

\section{Associate Editor: André Luiz Paranhos Perondini}

License information: This is an open-access article distributed under the terms of the Creative Commons Attribution License, which permits unrestricted use, distribution, and reproduction in any medium, provided the original work is properly cited. 